\title{
Zur Vereinfachung der röntgenologischen Fremdkörper-Lokalisation
}

(Kombination von Stereoskopie und Fürstenau-Tiefenbestimmung).

Von Prof. Dr. A. Köhler,

Stabsarzt d. L. am Reserve-Lazarett I, Wiesbaden.

Ebenso wie die große Anzahl der für eine Krankheit angegebenen therapeutischen Mittel bekanntlich beweist, daB keines von diesen Mitteln hinsichtlich des Erfolges den Arzt befriedigt, so besagt uns die Unmenge von mehreren Hundert röntgenologischen Fremdkörper-Lokalisationsmethoden, daß keine einzige von ihnen dem Untersucher in jedem Falle genügt. Die einfachen Methoden arbeiten nicht auf den Millimeter genau, und die komplizierten Methoden leiden daran, daß niemand eine Reihe solcher Methoden zu erlernen Zeit findet. Eine komplizierte Methode aber, die an Sicherheit alle anderen um ein so Bedeutendes überragt, daB sie jeder gewissenhafte Chirurg im Interesse seiner Kranken sich aneignen müßte, gibt es bis jetzt nicht. Von den bis jetzt bekannten komplizierten Methoden wird sich auch wohl im Laufe der Zeit keine als in .jeder Hinsicht beste herausheben. Jede der angegebenen umständlichen Methoden wird in der Regel nur von ihrem Erfinder, der sie natürlich immer als höchst einfach und nur scheinbar kompliziert hinstellt, und seinen Schülern geübt. . Sicher verwenden $95 \%$ der Untersucher noch die einfachen Methoden oder sind wieder zu ihnen zurückgekehrt. So habe ich vor $17 \mathrm{Jahren}$ in der Friedensröntgenologie 
mit den einfachen Methoden angefangen zu arbeiten, habe nach dem russisch-japanischen und dem türkisch-italienischen Kriege in der Heimat und jetzt seit dem ersten Kriegsmonat fast täglich Projektile nach mehreren Methoden bis zu den kompliziertesten gesucht, um in den letzten Monaten wieder zu den allereinfachsten Methoden zurückgekehrt zu sein Aufnahmen in zwei zueinander senkrechten Richtungen, stereoskopische Aufnahmen und Fürstenau-Methode, wenn auch mit kleinen Modifikationen. Die Anwendung der einfachsten Methoden, mögen sie auch um ein paar Millimeter ungenauer sein - der Chirurg mißt in der Wunde auch nicht mit dem Millimetermaß - hat außer dem Vorteil des energetischen Imperativs noch den ubiquitärer Verständlichkeit und Nachprüfungsmöglichkeit jedes Nachuntersuchers. Wie oft kommt es vor, da: ein Soldat operiert wird, ohne daß der Röntgenologe, der die Tiefenbestimmung ausgeführt, anwesend sein kann; oder daß die Operation die nach der Lokalisation geplant, aus äußeren Gründen zunächst um ein paar Tage verschoben und der Patient plötzlich in ein anderes Lazarett an einem anderen Ort überführt wird. Da muß der zweite Arzt aus der Krankengeschichte und den Plattenabzügen ohne unnötigen Zeitverlust ersehen können, wie und wo der Fremdkörper vom ersten Arzt gefunden worden ist (und eine kürzeste Schirmuntersuchung hat nur noch $\mathrm{zu}$ eruieren, ob der Fremdkörper eventuell inzwischen gewandert ist, was unter Hunderten vion Fällen höchstens einmal vorkommt). Bei komplizierten $\mathrm{Me}$ thoden aber muß der zweite Untersucher, der in der Methode des ersten nicht beschlagen ist, wieder von vorn anfangen mit der Lokalisation. Würde das zwar insofern nichts schaden, als durch die Kontrolle des zweiten bei Bestätigung der Richtigkeit der ersten Untersuchung das Resultat um so sicherer dasteht, so würde doch der Patient auf diese Weise der Verbrennungsgefahr eher ausgesetzt sein; hingegen. wenn eine einfache Methode durch eine andere einfache Methode nachgeprüft wird, so ist das in jedem Falle, zum mindesten himsichtlich Zeitverlustes, immer rationeller als bei $\mathrm{zw}$ ei komplizierten Methoden.

Aus allen diesen Gründen wird man wohl immer wieder auf die einfachen Methoden zurückkommen. Und um die Grobheit der einfachen Methoden wettzumachen, hindert nichts, daß man zwei der allereinfachsten Methoden in einer Handlung kombiniert und $\mathrm{daB}$ so gewissermaßen die eine Methode die andere ergänzt und auf ihre Richtigkeit kontrolliert. So kann man bei Extremitätenuntersuchungen nach kurzer Orientierung am Schirm und Anbringen von ein oder zwei Bleimarken auf der Haut eine einfache Profilaufnahme und dazu eine stereoskopische Dorsalaufnahme machen; oder am Rumpfe, Kopf, Hals und Hüfte fertigt man eine Fürstenau-Platte an und außerdem eine Stereoskopuntersuchung. Doch wird hierbei die militärische Oekonomie schlecht gewahrt. Nun genügt mir, wenn ich selbst operieren müßte, die einfache Fürstenau-Methode, allerdings mit gleichzeitiger Markierung des Nahepunktes, in fast allen Fällen. Aber die Chirurgen wollen meist ein stereoskopisches Bild sehen. Um nun den Chirurgen ein plastisches Bild zu vorschaffen, gleichzeitig dabei die einfache Fürstenausche Tiefenbestimmung zu erhalten und doch eine Platte zu sparen, mache ich in diesen Fällen eine stereoskopische Aufnahme im Fürstenau-Abstand von $60 \mathrm{~cm}$, nachdem kurz vorher am Schirm die plattennahe Hautstelle mit viereckiger Bleimarke bezeichnet ist, durch welche der auch den Fremdkörper schneidende Normalstrahl geht. Diese Bleimarke ist unerläBlich, weil sonst an den rein stereoskopischen Aufnahmen die Verschiebung des Fremdkörperschattens kaum gemessen werden kann. Diese Markierung, die bei gewissen primitiven Fluoreszenzschirmgestellen sehr schwierig sein mag, ist das Werk eines Augenblicks bei Verwendung der Bucky-Wabenblende, (meines Teleröntgenographengestells mit Bucky-Blende oder des Orthodiagraphen). Man kennzeichne sich nur durch irgendein Metallzeichen das mittlere Quadrat der Wabenblende. Wenn die Wabenblende richtig zentriert angebracht ist (anders ist sie ja überhaupt nicht zu gebrauchen), dann bringe man, z. B. bei Fremdkörpern im Rumpfe, bei Parallelismus des Schirms mit der Frontalebene des Rumpfes den Fremdkörperschatten zur Deckung mit dem Metallzeichen des mittelsten Bucky* Blendenquadrats; der Fremdkörper steht jetzt im Normalstrahl, und die in letzterem stehende plattennahe Hautstelle wird mit eckiger (nicht runder!) Bleimarke beklebt, die bis zur photographischen Aufnahme bleibt und dann mit Höllenstein o. ä. gekennzeichnet wird. Dann drehe man den Patienten um $180^{\circ}$ und markiere auch den Gegenpunkt, entweder mit eckiger Bleimarke oder nur mit Blaustift. Lag, der Schärfe nach beurteilt, das Corpus alienum mehr nach vorn bauchwärts, dann wird die Aufnahme in Bauchlage gemacht; die Bleimarke liegt der Platte auf, und die Röntgenröhre wird in Fürstenau-Abstand von $60 \mathrm{~cm}$ zunächst senkrecht über dem Gegenpunkt (auf dem Rücken des Patienten) angebracht und dann mit Verschiebung der Röhre nach rechts und links um $6,5 \mathrm{~cm}$ im ganzen eine regelrechte stereoskopische Aufnahme in Bartholdy-Kassette oder anderer Stereoskopkassette hergestellt. Entwickelt und getrocknet hat man also jetzt 1. eine plastische Aufnahme für Stereoskopbetrachtung, 2. die Möglichkeit, die Tiefenlage des Geschosses mit Fürstenau-Zirkel genau zu messen, 3. den Nahepunkt auf der Haut für zweckmäßiges chirurgisches Eingehen.
Zum Ueberfluß sei angegeben, obwohl es sich ohne weiteres ergibt, wie die Uebertragung der Bilder zur Fürstenau-Tiefenbestimmung geschieht: Man pause von der einen Nerv-Aufnahme den Bleimarkenschatten und den Fremdkörperschatten durch und lege diese Pause dann auf die andere Aufnahme, und zwar so, daß sich die Bleimarkenschatten genau decken; nun pause man den Fremdkörperschatten der zweiten Aufnahme dazu. Zwei identische Punkte der Fremdkörperschatten werden nun in die Oeffnung des Fürstenau-Zirkels genommen usw., wie bekannt. $\mathrm{Daß}$ für diesen Zweck die Bleimarke viereckig oder dreieckig sein muß und nicht kreisrund sein darf, ist klar, weil sonst beim Durchpausen der zweiten Aufnahme Verdrehungen möglich wären. - Sollte einmal während der Aufnahmen die der Haut aufgeklebte Bleimarke nicht direkt der Platte aufliegen können (z. B. bei größerer Lendenlordose, bei stark gekrümmtem Nacken usw.), sondern von der Platte 1, 2 oder $3 \mathrm{~cm}$ abstehen, so zeichne man beim Durchpausen der fertigen Platten die Schatten der Bleimarke nur so viel in der Horizontale auseinander, als der S pitzenabstand im Fürstenau-Zirkel bei Einstellung von 1,2 oder $3 \mathrm{~cm}$ der Skala "Tiefenlage" anzeigt, und ziehe schließlich, wenn man den Abstand des Geschosses von der Platte gefunden, die 1, 2 oder $3 \mathrm{~cm}$ wieder ab, um den Abstand des Geschosses von der Haut zu erhalten.

Zur bequemeren Ausführung der Stereoskopie habe ich mir vor vielen Jahren eine Bartholdv-Kassette nur in Größe 24/30 anfertigen lassen. Diese ist ungemein handlich und genügt für etwa. $95 \%$ aller Fremdkörperfälle.

Zusammenfassung. Wir kombinieren also hier $\mathrm{zwei}$ der allereinfachsten, jedem Röntgenologen und Chirurgen bekannten Lokalisationsarten zu einer Methode, die plastisches Sehen der Fremdkörperlage, Ausmessung der Tiefenlage in Zentimetern, Anhaltspunkte für chirurgisches Eingehen und militärische Sparsamkeit in sich vereinigt. Voraussetzung dabei ist nur, da $B$ man versteht, 1. einen Fremdkörper am Durchleuchtungsschirm in den Normalstrahl zu bringen (was bei Besitz einer Wabenblende besonders leicht und schnell geschieht), 2. stereoskopische Aufnahmen anzufertigen, 3. die einfache Tiefen bestimmungsmethode nach $F$ ürstenau auszuführen. 